\title{
Molecular differences between ductal carcinoma in situ and adjacent invasive breast carcinoma: A multiplex ligation-dependent probe amplification study
}

\author{
Cathy B. Moelans *, Roel A. de Weger, Hanneke N. Monsuur, Anoek H.J. Maes and Paul J. van Diest \\ Department of Pathology, University Medical Center Utrecht, Utrecht, The Netherlands
}

\begin{abstract}
Ductal carcinoma in situ (DCIS) accounts for approximately $20 \%$ of mammographically detected breast cancers. Although DCIS is generally highly curable, some women with DCIS will develop life-threatening invasive breast cancer, but the determinants of progression to infiltrating ductal cancer (IDC) are largely unknown. In the current study, we used multiplex ligation-dependent probe amplification (MLPA), a multiplex PCR-based test, to compare copy numbers of 21 breast cancer related genes between laser-microdissected DCIS and adjacent IDC lesions in 39 patients. Genes included in this study were ESR1, EGFR, FGFR1, ADAM9, IKBKB, PRDM14, MTDH, MYC, CCND1, EMSY, CDH1, TRAF4, CPD, MED1, HER2, CDC6, TOP2A, MAPT, BIRC5, CCNE1 and AURKA.

There were no significant differences in copy number for the 21 genes between DCIS and adjacent IDC. Low/intermediategrade DCIS showed on average 6 gains/amplifications versus 8 in high-grade DCIS $(p=0.158)$. Furthermore, alterations of AURKA and CCNE1 were exclusively found in high-grade DCIS, and HER2, PRDM14 and EMSY amplification was more frequent in high-grade DCIS than in low/intermediate-grade DCIS. In contrast, the average number of alterations in low/intermediate and high-grade IDC was similar, and although EGFR alterations were exclusively found in high-grade IDC compared to low/intermediate-grade IDC, there were generally fewer differences between low/intermediate-grade and high-grade IDC than between low/intermediate-grade and high-grade DCIS.

In conclusion, there were no significant differences in copy number for 21 breast cancer related genes between DCIS and adjacent IDC, indicating that DCIS is genetically as advanced as its invasive counterpart. However, high-grade DCIS showed more copy number changes than low/intermediate-grade DCIS with specifically involved genes, supporting a model in which different histological grades of DCIS are associated with distinct genomic changes that progress to IDC in different routes. These high-grade DCIS specific genes may be potential targets for treatment and/or predict progression.
\end{abstract}

Keywords: DCIS, IDC, MLPA, laser microdissection, breast cancer

\section{Introduction}

The most widely held concept regarding the development of human breast cancer suggests that breast cancer progression is a multistep process that manifests itself as a sequence of pathologically defined

\footnotetext{
*Corresponding author: Cathy B. Moelans, PhD, Department of Pathology (H04.312), University Medical Center Utrecht, Heidelberglaan 100, P.O. Box 85500, 3508GA Utrecht, The Netherlands. Tel.: +31 88 7557617; Fax: +31 30 2544990; E-mail: cmoelans@umcutrecht.nl.
}

stages with ductal carcinoma in situ (DCIS) as final pre-invasive stage before progression to invasive ductal carcinoma (IDC). Pathologically, DCIS is a clonal epithelial proliferation that does not breech the basement membrane. IDC, on the other hand, is an abnormal proliferation of breast epithelial cells that infiltrates through the basement membrane into the surrounding stroma. The proportion of patients diagnosed with DCIS, and with a mixture of DCIS and IDC, is increasing as screening mammography becomes more common. DCIS now accounts for approximately $20 \%$ of mammographically detected breast cancers. Some 
women with DCIS will develop life-threatening invasive breast cancer, but the determinants of progression to IDC are largely unknown. Therefore, all women with DCIS are generally treated fairly aggressively with surgery and radiation. Biologic markers that predict recurrence and/or progression to invasive cancer other than traditional grading are thus warranted.

In the most popular model to explain the development of IDC, low-grade DCIS tends to progress to low-grade IDC, and high-grade DCIS tends to progress to high-grade IDC by accumulation of fairly specific chromosomal and gene alterations [5,6,23,24,37]. In the last decades, much progress has been made in understanding the molecular and genetic events that underlie the transition from preinvasive lesions such as DCIS to IDC. The majority of molecular changes that are observed in breast cancer seem to be already evident in the DCIS stage $[33,36]$. The search for biologic markers that determine the transition from DCIS to IDC and thereby predict the natural course of DCIS is, however, ongoing.

An impediment to our understanding of the biological course of DCIS has been the limited extent of DCIS in clinical specimens. Laser capture microdissection (LCM) has, however, enabled the acquisition of pure populations of target cells and has proven to be popular for use in comparative genomic hybridization (CGH) [2] and loss of heterozygosity ( $\mathrm{LOH}$ ) studies [4]. Still, the amount of DNA that can be isolated in this way is often a limiting factor. In contrast with other high throughput analysis methods, multiplex ligation-dependent probe amplification (MLPA) requires only small quantities of short DNA fragments, which makes it very suitable for analysis of microdissected paraffin embedded material [30]. In previous studies we used MLPA to detect HER2 [28,30] and TOP2A [31] amplification and used its multiplex aspect to determine the frequency of polysomy 17 in breast tumors [32].

In the current study, we applied MLPA to compare copy number changes in 21 breast cancer related genes between laser-microdissected DCIS and adjacent IDC lesions in 39 patients.

\section{Materials and methods}

\subsection{Patient material}

From paraffin embedded breast cancer resection specimens of 39 patients, tissue blocks with DCIS and adjacent IDC were selected by an experienced pathologist (PJvD) based on the H\&E stained sections. Grading of DCIS and invasive cancer was done according to usual methods [16,20]. DCIS and IDC lesions were subdivided into low/intermediate-grade DCIS/IDC (DCIS $n=19$ and IDC $n=15$ ) and highgrade DCIS/IDC (DCIS $n=20$ and IDC $n=24$ ). Grading was done independently by two pathologists and discordances were discussed until consensus was reached. Anonymous use of redundant tissue for research purposes is part of the standard treatment agreement with patients in our hospital [46].

\subsection{Laser microdissection}

Laser microdissection was performed on $8 \mu \mathrm{m}$ thick paraffin sections (by comparing with a serial $\mathrm{H} \& \mathrm{E}$ stained slide where IDC and DCIS were marked). For laser microdissection, sections were baked at $56^{\circ} \mathrm{C}$ for $1 \mathrm{~h}$, deparaffinized in xylene for $10 \mathrm{~min}$ and rehydrated through graded alcohols $(100 \%, 85 \%$ and $70 \%$ for $1 \mathrm{~min}$ each). After staining with haematoxylin for $5 \mathrm{~s}$, slides were rinsed in water and dipped in eosin for 5 s. Finally, slides were dehydrated in $100 \%$ ethanol for $1 \mathrm{~min}$ and air dried. At this point Liquid Cover Glass (PALM AG, Bernried, Germany) was applied by aerosol to improve morphology and to allow larger tissue areas to be laser pressure-catapulted, and sections were air dried for at least $30 \mathrm{~min}$. A PALM microdissection system with UV laser was used to separate between 4 and $20 \mathrm{~mm}^{2}$ of DCIS or IDC from their surrounding tissue. Subsequently, these areas were catapulted by laser pressure catapulting into a cap of a common microfuge tube moistened with a drop of mineral oil.

\subsection{Multiplex ligation-dependent probe amplification (MLPA)}

After laser microdissection, DNA was isolated by $1 \mathrm{~h}$ incubation in proteinase $\mathrm{K}(10 \mathrm{mg} / \mathrm{ml}$; Roche, Almere, The Netherlands) at $56^{\circ} \mathrm{C}$ followed by boiling for $10 \mathrm{~min}$. This DNA solution $(50 \mu \mathrm{l})$ was, after centrifugation, used in the MLPA analysis according the manufacturers' instructions, using the P078-B1 breast kit (MRC Holland, Amsterdam, The Netherlands). Table 1 shows the contents of this kit and includes chromosomal locations of all probes. All tests were per- 
Table 1

Contents of the P078-B1 MLPA kit (MRC Holland, The Netherlands)

\begin{tabular}{|c|c|c|c|c|c|}
\hline Gene & Chr & Mapview position & No. probes & Transcript description & Ref. \\
\hline \multirow[t]{2}{*}{ ESR1 } & $06 \mathrm{q} 25$ & $06-152.423838$ & 2 & Transcription factor & {$[21]$} \\
\hline & & $06-152.457215$ & & & \\
\hline \multirow[t]{2}{*}{$E G F R$} & $07 \mathrm{p} 11$ & $07-055.191055$ & 2 & Receptor tyrosine kinase involved in signal transduction & [39] \\
\hline & & 07-055.233957 & & & \\
\hline \multirow[t]{2}{*}{ FGFR1 } & $08 \mathrm{p} 12$ & $08-038.391533$ & 2 & Receptor tyrosine kinase involved in signal transduction & {$[11,27]$} \\
\hline & & 08-038.434092 & & & \\
\hline$A D A M 9$ & $08 \mathrm{p} 11$ & 08-038.998319 & 1 & Metalloproteinase associated with protein metabolism & {$[11,27]$} \\
\hline \multirow[t]{2}{*}{$I K B K B$} & $08 \mathrm{p} 11$ & 08-042.292902 & 2 & Serine/threonine kinase associated with signal transduction & [11] \\
\hline & & 08-042.302676 & & & \\
\hline PRDM14 & $08 \mathrm{q} 13$ & 08-071.130073 & 1 & Transcription regulatory protein & {$[35]$} \\
\hline \multirow[t]{2}{*}{$M T D H$} & $08 \mathrm{q} 22$ & 08-098.742504 & 2 & Metastasis promoting gene involved in chemoresistence & {$[22]$} \\
\hline & & 08-098-788082 & & & \\
\hline \multirow[t]{3}{*}{$M Y C$} & $08 \mathrm{q} 24$ & $08-128.821796$ & 3 & Transcription factor involved in apoptosis and cell proliferation & [42] \\
\hline & & 08-128.822001 & & & \\
\hline & & $08-128.822151$ & & & \\
\hline \multirow[t]{2}{*}{ CCND1 } & $11 \mathrm{q} 13$ & $11-069.167779$ & 2 & Cell cycle control protein involved in signal transduction & [26] \\
\hline & & $11-069.175089$ & & & \\
\hline \multirow[t]{2}{*}{ EMSY } & $11 \mathrm{q} 13$ & $11-075.902087$ & 2 & Transcription regulatory protein & [26] \\
\hline & & $11-075.926543$ & & & \\
\hline \multirow[t]{2}{*}{$\mathrm{CDH1}$} & $16 \mathrm{q} 22$ & $16-067.328716$ & 2 & Adhesion molecule associated with signal transduction & {$[12]$} \\
\hline & & $16-067.404826$ & & & \\
\hline TRAF4 & $17 q 11$ & $17-024.098403$ & 1 & $\begin{array}{l}\text { Adaptor molecule involved in signal } \\
\text { transduction, cell proliferation and apoptosis }\end{array}$ & {$[10]$} \\
\hline$C P D$ & $17 q 11$ & $17-025.795018$ & 1 & Carboxypeptidase involved in protein metabolism & - \\
\hline MED1 & $17 q 12$ & $17-034.840858$ & 1 & Transcription regulatory protein involved in signal transduction & [48] \\
\hline \multirow[t]{4}{*}{$H E R 2$} & $17 q 12$ & $17-035-118101$ & 4 & Receptor tyrosine kinase associated with signal transduction & {$[30]$} \\
\hline & & $17-035.127183$ & & & \\
\hline & & $17-035-133169$ & & & \\
\hline & & $17-035.136344$ & & & \\
\hline CDC6 & $17 q 21$ & $17-035.699283$ & 1 & Cell cycle control protein involved in signal transduction & [1] \\
\hline \multirow[t]{3}{*}{ TOP $2 A$} & $17 q 21$ & $17-035.812698$ & 3 & DNA topoisomerase protein involved in & [38] \\
\hline & & $17-035.816651$ & & regulation of the topological status of DNA & \\
\hline & & $17-035.818297$ & & & \\
\hline$M A P T$ & $17 q 21$ & $17-041.423085$ & 1 & Structural protein involved in cell growth and/or maintenance & - \\
\hline \multirow[t]{3}{*}{ BIRC5 } & $17 q 25$ & $17-073.722036$ & 3 & Adapter molecule involved in signal & {$[15]$} \\
\hline & & $17-073.722396$ & & transduction, cell communication and cell & \\
\hline & & $17-073.724340$ & & survival & \\
\hline \multirow[t]{2}{*}{ CCNE1 } & $19 \mathrm{q} 12$ & $19-035.000150$ & 2 & Cell cycle control protein involved in signal transduction & {$[9,25]$} \\
\hline & & $19-035.005214$ & & & \\
\hline$A U R K A$ & $20 q 13$ & $20-054.389980$ & 1 & Serine/threonine kinase involved in signal transduction & {$[18,43]$} \\
\hline
\end{tabular}

Note: For each gene the chromosomal position, mapview distance from $p$-telomere, the number of probes present in the MLPA kit, a description of the transcript protein and if possible a relevant (breast cancer) reference is given.

formed in duplicate on an ABI 9700 PCR machine (Applied Biosystems, Foster City, CA, USA). PCR products were analyzed on an ABI310 capillary sequencer (Applied Biosystems). Gene copy numbers were analyzed using Genescan (Applied Biosystems) and Coffalyser (version 7.0) software (MRC-Holland). Four negative reference samples (normal breast) were taken along in each MLPA run to normalize MLPA ratios. For genes with more than one probe present in the kit, the mean of all the probe peaks of this gene in 
duplicate was calculated. A mean value below 0.7 was defined as loss, a value between $0.7-1.3$ was defined as normal, $1.3-2.0$ as gain and values $>2.0$ were defined as (high level) amplification, as established previously $[7,14]$.

\subsection{Statistics}

Statistics were performed using SPSS statistical software. Copy number ratio differences for the 21 individual genes between DCIS and adjacent IDC cases were compared with paired $t$-test. In addition, we compared the mean copy number ratio of each individual gene between the grouped DCIS cases on the one hand and the IDC cases on the other. Next, MLPA data were dichotomized as non-amplified vs. gain/amplified (cut-off 1.3), and these gene dosage categories were compared between DCIS and IDC by McNemar's test. Furthermore, the mean MLPA copy number ratio including all 21 genes in all 39 patients was compared between DCIS and IDC by $t$-test.

Hereafter, we compared different grades of DCIS and IDC. First, we compared the mean copy number ratios between low/intermediate and high-grade DCIS, as well as between low/intermediate-grade and highgrade IDC by $t$-test. Then, we again dichotomized data and compared the mean number of gains/amplifications between low/intermediate and high-grade DCIS, as well as between low/intermediate-grade and highgrade IDC by $t$-test. $p$-values below 0.05 were con- sidered significant. Bonferroni correction was used to address the problem of multiple comparisons. Finally, unsupervised hierarchical clustering was applied with $\mathrm{R}$ as before [32].

\section{Results}

Supplementary Table 1 (Suppl. Table 1: http://www. qub.ac.uk/isco/JCO/) shows the raw copy number data. Paired comparison of copy number ratios between DCIS and adjacent invasive lesions did not yield significance for any of the tested genes. Overall (unpaired) comparison of copy number ratios between the grouped DCIS and invasive lesions also did not yield significance for any of the tested genes.

Figure 1 shows the frequency of gain/amplification of 21 genes analyzed by MLPA in DCIS and adjacent IDC, using dichotomized data. Although BIRC5 (survivin), TOP2A, CCND1 and MED1 (PPARBP) gain/amplification seemed to be more prevalent in DCIS than IDC, overall, there were no significant differences between both components. Chromosomes 8 , 11 and 17 seem to show the most frequent alterations in both breast lesions, the analyzed regions on chromosomes $6,7,16,19$ and 20 to a lesser extent. We found three genes showing frequent $(>5 \%)$ loss: FGFR1, CDH1 and MAPT, with also no significant differences between DCIS and IDC.

\section{$\square$ DCIS amplifications (ratio > 1.3) $\square$ IDC amplifications (ratio > 1.3)}

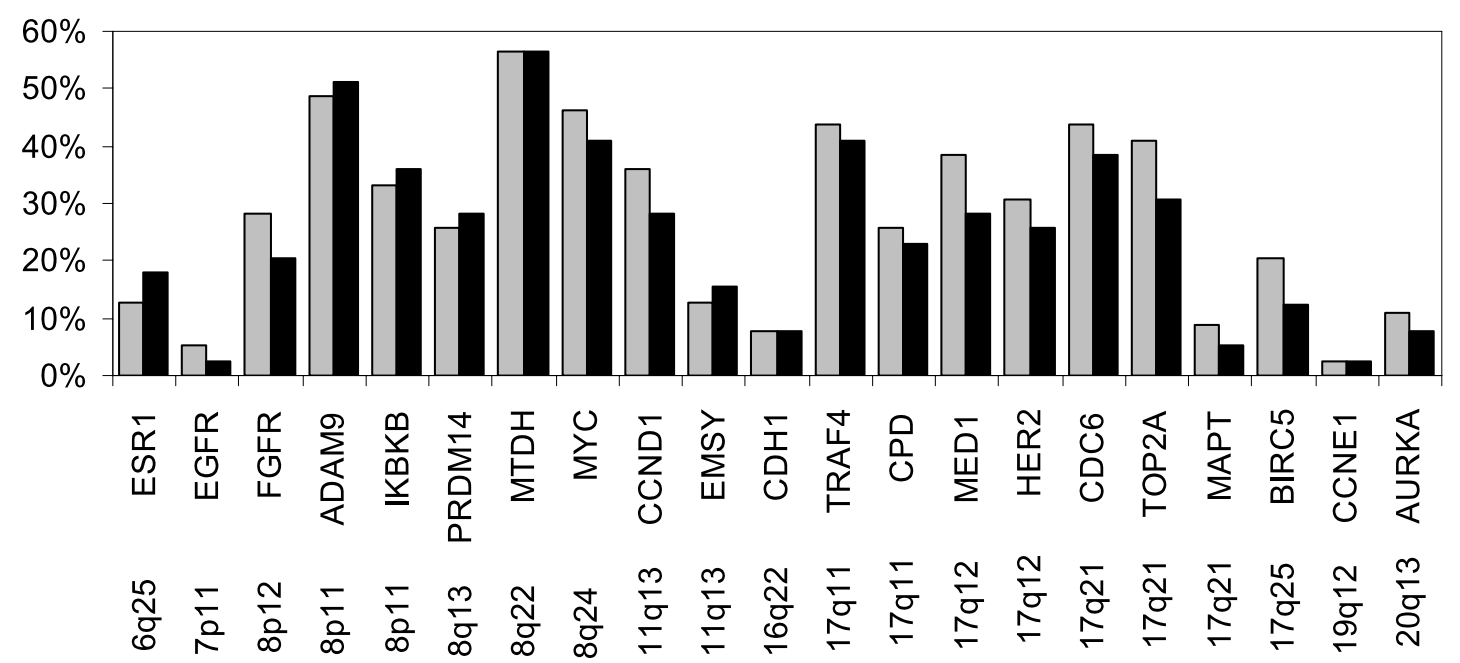

Fig. 1. Amplification frequencies of 21 genes analyzed by multiplex ligation-dependent probe amplification (MLPA) in DCIS and adjacent IDC from 39 breast cancer patients. 
Overall, there was no significant copy number difference between DCIS $(1.35 \pm 0.85)$ and IDC $(1.34 \pm$ 0.88) $(p=0.604)$.

The mean copy number ratio was $1.29 \pm 0.71$ in low/intermediate-grade and $1.42 \pm 0.96$ in high-grade DCIS ( $p=0.023)$, and $1.30 \pm 0.71$ in low/intermediate-grade and $1.36 \pm 0.97$ in high-grade IDC ( $p=$ 0.308 ). The mean number of gains/amplifications in the 21 analyzed genes was 6 in low/intermediate-grade DCIS and 8 in high-grade DCIS ( $p=0.158)$, and 6 in both low/intermediate-grade and high-grade IDC $(p=0.903)$.

Also, as shown in Table 2, the pattern of alterations differed between low/intermediate-grade and high-grade DCIS, and in a much lesser extent between low/intermediate and high-grade IDC. Alterations of AURKA and CCNE1 were exclusively found in highgrade DCIS, and alterations of EGFR were exclusively found in high-grade IDC. Figure 2 shows that gene copy numbers of HER2 $(p=0.013)$, MYC $(p=$ 0.009 ) and MTDH (not significant) were higher in high-grade DCIS compared to low/intermediate-grade DCIS. Copy numbers of HER2 $(p=0.037)$ were also significantly higher in high-grade IDC compared to low/intermediate-grade IDC, as were those of TRAF4 $(p=0.088)$, but MAPT $(p=0.108)$ and CCNE1 $(p=0.070)$ gene copy numbers seemed to be lower in high-grade IDC compared to low-grade IDC.

Figure 3 shows the cluster analysis for all 39 DCIS/IDC pairs according to their MLPA profiles based on 21 genes and their grading. Twenty of the 39 pairs of DCIS/IDC $(51 \%)$ clustered closely together. Although not consistently, most of the low/intermediate-grade DCIS/IDC seemed to belong to a different cluster than high-grade DCIS/IDC.

\section{Discussion}

This study aimed to investigate and compare copy number changes within DCIS and adjacent IDC by MLPA. Overall, there was no significant copy number difference between DCIS and IDC, and the detected genetic alterations in tumors with synchronous DCIS and invasive ductal cancer showed a high degree of shared changes within the two components.

Table 2

Frequencies of alterations (gain, amplification and loss) in low/intermediate-grade and high-grade DCIS and adjacent IDC present in 39 breast tumor samples

\begin{tabular}{|c|c|c|c|c|}
\hline & \multicolumn{2}{|l|}{ DCIS } & \multicolumn{2}{|l|}{ IDC } \\
\hline & Low/intermediate-grade & High-grade & Low/intermediate-grade & High-grade \\
\hline$\overline{\text { ESR1 }}$ & 16 & 15 & 13 & 25 \\
\hline EGFR & 5 & 10 & 0 & 4 \\
\hline FGFR1 & 32 & 55 & 27 & 38 \\
\hline ADAM9 & 58 & 45 & 67 & 50 \\
\hline IKBKB & 37 & 40 & 47 & 38 \\
\hline PRDM14 & 11 & 40 & 33 & 29 \\
\hline MTDH & 53 & 65 & 47 & 83 \\
\hline MYC & 42 & 65 & 47 & 63 \\
\hline CCND1 & 53 & 40 & 27 & 42 \\
\hline EMSY & 11 & 20 & 33 & 33 \\
\hline CDH1 & 21 & 15 & 7 & 25 \\
\hline TRAF4 & 42 & 65 & 33 & 58 \\
\hline CPD & 21 & 40 & 53 & 33 \\
\hline MED1 & 42 & 50 & 40 & 29 \\
\hline HER 2 & 21 & 65 & 33 & 33 \\
\hline CDC6 & 42 & 55 & 47 & 42 \\
\hline TOP2A & 47 & 45 & 47 & 29 \\
\hline MAPT & 16 & 35 & 7 & 21 \\
\hline BIRC5 & 26 & 30 & 13 & 13 \\
\hline CCNE1 & 0 & 15 & 7 & 4 \\
\hline AURKA & 0 & 25 & 7 & 8 \\
\hline
\end{tabular}

Notes: DCIS - ductal carcinoma in situ; IDC - invasive ductal carinoma. 
HER2

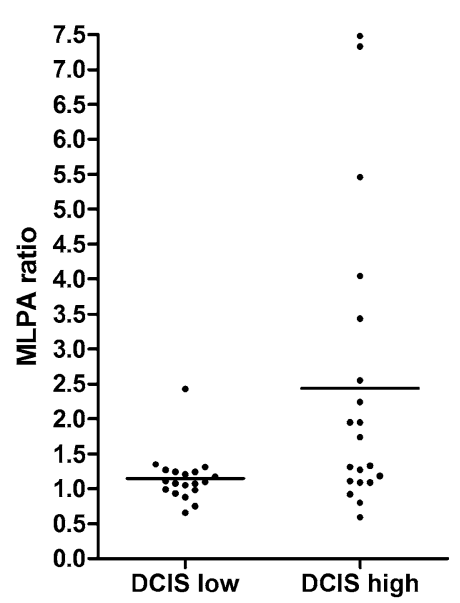

MYC

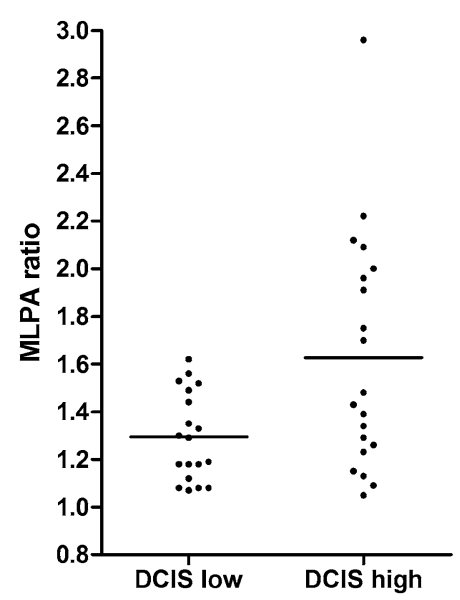

MTDH

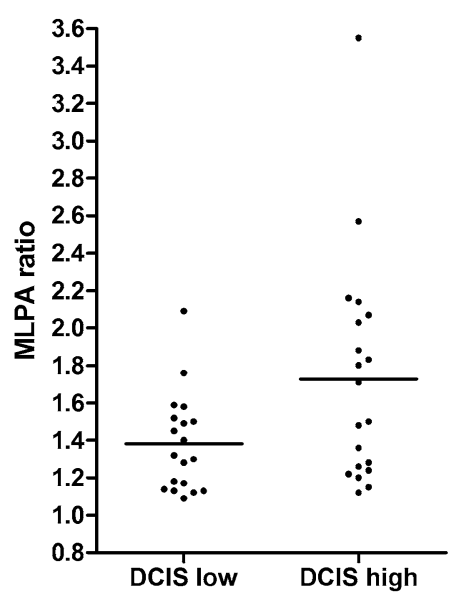

Fig. 2. MLPA copy number ratio's of HER2, MYC and MTDH in low/intermediate-grade DCIS (DCIS low, $n=19$ ) and high-grade DCIS (DCIS high, $n=20$ ) in 39 breast tumors.
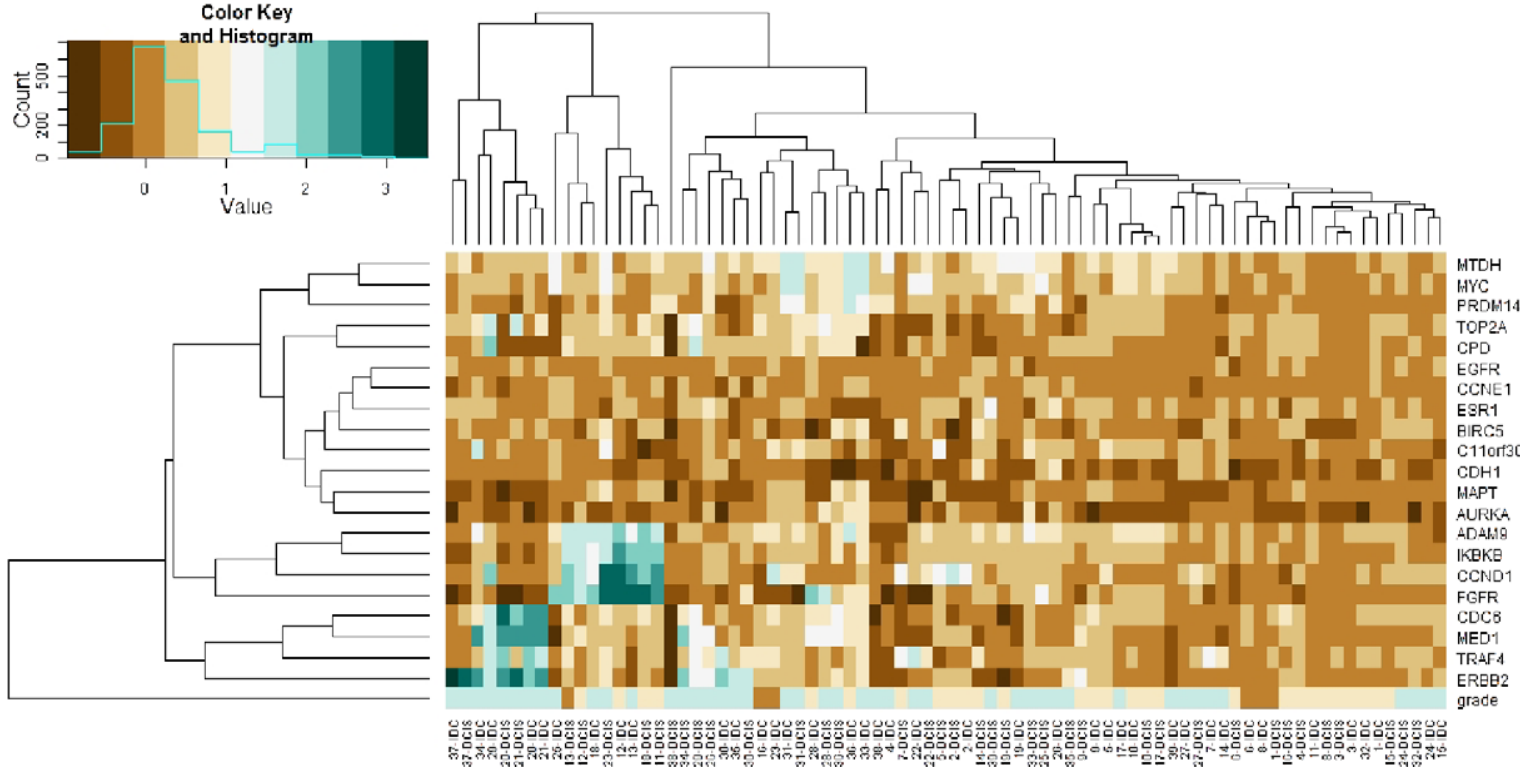

Fig. 3. Cluster analysis of 21 genes in low/intermediate and high-grade DCIS and adjacent IDC (Grade: dark brown: grade 1, light brown: grade 2, blue: grade 3). (The colors are visible in the online version of the article; http://dx.doi.org/10.3233/ACP-CLO-2010-0546.)

This is consistent with previous studies [23] and underlines that DCIS is genetically advanced, showing a similar degree and position of chromosomal alterations as invasive ductal cancer. Although copy number changes of some genes such as BIRC5 seemed to be more prevalent in DCIS than IDC, overall, no significant differences in gene copy number between DCIS and adjacent IDC were found. BIRC5 (survivin) has been described to be expressed in the full spectrum of breast tissue from normal to IDC [45]. A 3-fold higher cytoplasmic expression level has been described in DCIS compared to IDC which has been correlated with recurrence risk [3]. Our results also confirm previous studies showing amplification (and overexpression) of CCND1 in low-grade DCIS as well as in high-grade DCIS $[8,17,44]$. According to the author's current knowledge, many of the other genes investigated in this study, including MED1, FGFR1, IKBKB, PRDM14, MTDH, CPD, CCNE1 and AURKA have not been investigated for their copy number alterations 
in DCIS and adjacent IDC until now. A previous study has investigated copy number alterations of all genes in this study in a large group of IDC using the same MLPA kit and showed similar copy number alteration frequencies in literature [29].

The number as well as the pattern of alterations differed between low/intermediate and high-grade DCIS, supporting a model in which different histological grades of DCIS are associated with distinct genomic changes [13]. These genes showing alterations may be potential targets for treatment and/or markers of prognosis. HER2 showed more frequent gain/amplification in high-grade DCIS (65\%) than in low/intermediate $(21 \%)$, which is consistent with previous studies showing more frequent gain of $17 \mathrm{q}$ and higher HER 2 expression levels in high-grade DCIS [13,23,47]. Poorly differentiated DCIS has also been associated with frequent loss of $8 \mathrm{p}$, which is consistent with our data since FGFR1 on 8p11 showed no loss in low/intermediategrade DCIS compared to $25 \%$ loss in high-grade DCIS. MYC amplifications were found in IDC as well as in DCIS without any significant differences in frequency between both components. This is inconsistent with a FISH study that could not demonstrate MYC amplifications in DCIS adjacent to FISH-amplified IDC [41]. In this study, the MLPA ratio was higher in IDC compared to DCIS in $56 \%$ of cases, but in most cases $(16 / 22)$ this did not lead to a different amplification status (gain vs. normal, no differences were found in the 13\% high level amplifications) between both lesions. Furthermore, MYC amplifications seem to be more prevalent in high-grade DCIS lesions $(65 \%)$ than in low/intermediate-grade lesions (42\%), which is consistent with previous studies [8].

Some alterations (CCNE1 19q12 and AURKA 20q13) were exclusively found in high-grade DCIS. However, at this moment, no single gene has been identified that differentiates between different types of DCIS. Perhaps the indicated genes could be candidates. Given the small size of the present study group, these data have to be confirmed in larger studies.

Table 2 and statistical analysis show that, overall, the differences between low/intermediate and high-grade DCIS seemed to be bigger than between low/intermediate and high-grade IDC. For example, whereas HER2, EMSY and PRDM14 gains/amplifications were as frequent in low/intermediate-grade IDC as in highgrade IDC, the amplification of these genes seemed to be less frequent in low/intermediate-grade DCIS than in high-grade DCIS. These differences may be prognostically important, or may turn out to be important variables in the choice of therapy.
Cluster analysis showed a trend towards clustering by grade, but several low/intermediate-grade DCIS/ IDC clustered together with high-grade DCIS/IDC, indicating that low-grade DCIS can be genetically as advanced as high-grade DCIS. Twenty of the 39 DCISIDC pairs clustered closely together, indicating a similar genetic profile of both components. This implies that, at least for the studied genes, DCIS is genetically as advanced as its invasive counterpart. Nevertheless, although no significant differences were observed between DCIS and IDC, not all DCIS/IDC pairs clustered together. It is possible that a larger gene set spread over more different chromosomal locations could better discriminate the samples. For example, Hannemann et al. identified a gene expression classifier of 35 genes which differed between DCIS and invasive breast cancer [19]. This same group also identified a 43-gene classifier to separate well- and poorly-differentiated DCIS samples. Unfortunately, the genes identified in that study did not overlap with the genes investigated using the MLPA P078-B1 kit in our study, except for ESR1. This gene was identified by Hannemann et al. as a discriminator between well- and poorlydifferentiated DCIS. However, this gene did not show significant differences in our study $(15 \%$ and $16 \%$ alterations). This could be explained by the fact that Hannemann et al. looked at the expression of ESR 1 in contrast to the copy number. Several studies have indicated that there is no absolute relationship between ESR1 gain or amplification and mRNA and protein expression in breast cancer [34,40].

In conclusion, this study showed that MLPA is suited to simultaneously detect amplification or loss of many potential prognostic or predictive genes in breast cancer. We found no significant differences between DCIS and adjacent IDC, but the number as well as the pattern of alterations differed between highand low/intermediate-grade DCIS. Overall, the differences between low/intermediate and high-grade DCIS seemed to be bigger than between low/intermediate and high-grade IDC.

\section{References}

[1] E. Arriola, C. Marchio, D.S. Tan, S.C. Drury, M.B. Lambros, R. Natrajan et al., Genomic analysis of the HER2/TOP2A amplicon in breast cancer and breast cancer cell lines, Lab. Invest. 88 (2008), 491-503.

[2] M. Aubele, A. Mattis, H. Zitzelsberger, A. Walch, M. Kremer, G. Welzl et al., Extensive ductal carcinoma in situ with small foci of invasive ductal carcinoma: evidence of genetic resemblance by CGH, Int. J. Cancer 85 (2000), 82-86.

[3] N. Barnes, P. Haywood, P. Flint, W.F. Knox and N.J. Bundred, Survivin expression in in situ and invasive breast cancer relates 
to COX-2 expression and DCIS recurrence, Br. J. Cancer 94 (2006), 253-258.

[4] P. Bertheau, L.F. Plassa, F. Lerebours, A. de Roquancourt, E. Turpin, R. Lidereau et al., Allelic loss detection in inflammatory breast cancer: improvement with laser microdissection, Lab. Invest. 81 (2001), 1397-1402.

[5] H. Buerger, E.C. Mommers, R. Littmann, R. Simon, R. Diallo, C. Poremba et al., Ductal invasive G2 and G3 carcinomas of the breast are the end stages of at least two different lines of genetic evolution, J. Pathol. 194 (2001), 165-170.

[6] H. Buerger, R. Simon, K.L. Schafer, R. Diallo, R. Littmann, C. Poremba et al., Genetic relation of lobular carcinoma in situ, ductal carcinoma in situ, and associated invasive carcinoma of the breast, Mol. Pathol. 53 (2000), 118-121.

[7] D.J. Bunyan, D.M. Eccles, J. Sillibourne, E. Wilkins, N.S. Thomas, J. Shea-Simonds et al., Dosage analysis of cancer predisposition genes by multiplex ligation-dependent probe amplification, Br. J. Cancer 91 (2004), 1155-1159.

[8] L. Burkhardt, T.J. Grob, I. Hermann, E. Burandt, M. Choschzick, F. Janicke et al., Gene amplification in ductal carcinoma in situ of the breast, Breast Cancer Res. Treat. 123(3) (2010), 757-765.

[9] G. Callagy, P. Pharoah, S.F. Chin, T. Sangan, Y. Daigo, L. Jackson et al., Identification and validation of prognostic markers in breast cancer with the complementary use of array-CGH and tissue microarrays, J. Pathol. 205 (2005), 388-396.

[10] S. Camilleri-Broet, I. Cremer, B. Marmey, E. Comperat, F. Viguie, J. Audouin et al., TRAF4 overexpression is a common characteristic of human carcinomas, Oncogene 26 (2007), 142-147.

[11] K. Chin, S. DeVries, J. Fridlyand, P.T. Spellman, R. Roydasgupta, W.L. Kuo et al., Genomic and transcriptional aberrations linked to breast cancer pathophysiologies, Cancer Cell 10 (2006), 529-541.

[12] A.M. Cleton-Jansen, E-cadherin and loss of heterozygosity at chromosome 16 in breast carcinogenesis: different genetic pathways in ductal and lobular breast cancer?, Breast Cancer Res. 4 (2002), 5-8.

[13] J. Climent, J.L. Garcia, J.H. Mao, J. Arsuaga and J. PerezLosada, Characterization of breast cancer by array comparative genomic hybridization, Biochem. Cell Biol. 85 (2007), 497508 .

[14] J. Coffa, M.A. van de Wiel, B. Diosdado, B. Carvalho, J. Schouten and G.A. Meijer, MLPAnalyzer: data analysis tool for reliable automated normalization of MLPA fragment data, Cell. Oncol. 30 (2008), 323-335.

[15] L.M. Davis, C. Harris, L. Tang, P. Doherty, P. Hraber, Y. Sakai et al., Amplification patterns of three genomic regions predict distant recurrence in breast carcinoma, J. Mol. Diagn. 9 (2007), 327-336.

[16] C.W. Elston and I.O. Ellis, Pathological prognostic factors in breast cancer. I. The value of histological grade in breast cancer: experience from a large study with long-term follow-up, Histopathology 19 (1991), 403-410.

[17] M. Fiche, H. Avet-Loiseau, C.M. Maugard, C. Sagan, M.F. Heymann, M. Leblanc et al., Gene amplifications detected by fluorescence in situ hybridization in pure intraductal breast carcinomas: relation to morphology, cell proliferation and expression of breast cancer-related genes, Int. J. Cancer 89 (2000), 403-410.
[18] C. Ginestier, N. Cervera, P. Finetti, S. Esteyries, B. Esterni, J. Adelaide et al., Prognosis and gene expression profiling of 20q13-amplified breast cancers, Clin. Cancer Res. 12 (2006), 4533-4544.

[19] J. Hannemann, A. Velds, J.B. Halfwerk, B. Kreike, J.L. Peterse and M.J. van de Vijver, Classification of ductal carcinoma in situ by gene expression profiling, Breast Cancer Res. 8 (2006), R61.

[20] R. Holland, J.L. Peterse, R.R. Millis, V. Eusebi, D. Faverly et al., Ductal carcinoma in situ: a proposal for a new classification, Semin. Diagn. Pathol. 11 (1994), 167-180.

[21] F. Holst, P.R. Stahl, C. Ruiz, O. Hellwinkel, Z. Jehan, M. Wendland et al., Estrogen receptor alpha (ESR1) gene amplification is frequent in breast cancer, Nat. Genet. 39 (2007), 655-660.

[22] G. Hu, R.A. Chong, Q. Yang, Y. Wei, M.A. Blanco, F. Li et al., MTDH activation by $8 \mathrm{q} 22$ genomic gain promotes chemoresistance and metastasis of poor-prognosis breast cancer, Cancer Cell 15 (2009), 9-20.

[23] E.S. Hwang, S. DeVries, K.L. Chew, D.H. Moore, K. Kerlikowske, A. Thor et al., Patterns of chromosomal alterations in breast ductal carcinoma in situ, Clin. Cancer Res. 10 (2004), 5160-5167.

[24] T. Irvine and I.S. Fentiman, Biology and treatment of ductal carcinoma in situ, Expert Rev. Anticancer Ther. 7 (2007), 135145.

[25] L.B. Jensen, J.M. Bartlett, C.J. Witton, T. Kirkegaard, S. Brown, S. Muller et al., Frequent amplifications and deletions of G1/S-phase transition genes, CCND1 and MYC in early breast cancers: a potential role in G1/S escape, Cancer Biomark. 5 (2009), 41-49.

[26] T. Kirkegaard, K.V. Nielsen, L.B. Jensen, F.M. Campbell, S. Muller, S.M. Tovey et al., Genetic alterations of CCND1 and EMSY in breast cancers, Histopathology 52 (2008), 698-705.

[27] S.S. Kwek, R. Roy, H. Zhou, J. Climent, J.A. MartinezCliment, J. Fridlyand et al., Co-amplified genes at 8p12 and $11 \mathrm{q} 13$ in breast tumors cooperate with two major pathways in oncogenesis, Oncogene 28 (2009), 1892-1903.

[28] C.B. Moelans, R.A. de Weger, C. Ezendam and P.J. van Diest, HER-2/neu amplification testing in breast cancer by Multiplex Ligation-dependent Probe Amplification: influence of manualand laser microdissection, BMC Cancer 9 (2009), 4.

[29] C.B. Moelans, R.A. de Weger, H.N. Monsuur, R. Vijzelaar and P.J. van Diest, Molecular profiling of invasive breast cancer by multiplex ligation-dependent probe amplification-based copy number analysis of tumor suppressor and oncogenes, Mod. Pathol. 23 (2010), 1029-1039.

[30] C.B. Moelans, R.A. de Weger, M.T. van Blokland, C. Ezendam, S. Elshof, M.G. Tilanus et al., HER-2/neu amplification testing in breast cancer by multiplex ligation-dependent probe amplification in comparison with immunohistochemistry and in situ hybridization, Cell. Oncol. 31 (2009), 1-10.

[31] C.B. Moelans, R.A. de Weger, M.T. van Blokland, E. van der Wall and P.J. van Diest, Simultaneous detection of TOP2A and HER2 gene amplification by multiplex ligation-dependent probe amplification in breast cancer, Mod. Pathol. 23 (2010), 62-70.

[32] C.B. Moelans, R.A. de Weger and P.J. van Diest, Absence of chromosome 17 polysomy in breast cancer: analysis by CEP17 chromogenic in situ hybridization and multiplex ligation- 
dependent probe amplification, Breast Cancer Res. Treat. 120 (2010), 1-7.

[33] E.C. Mommers, A.M. Leonhart, F. Falix, R. Michalides, C.J. Meijer, J.P. Baak et al., Similarity in expression of cell cycle proteins between in situ and invasive ductal breast lesions of same differentiation grade, J. Pathol. 194 (2001), 327-333.

[34] R.M. Neve, K. Chin, J. Fridlyand, J. Yeh, F.L. Baehner, T. Fevr et al., A collection of breast cancer cell lines for the study of functionally distinct cancer subtypes, Cancer Cell 10 (2006), 515-527.

[35] N. Nishikawa, M. Toyota, H. Suzuki, T. Honma, T. Fujikane, T. Ohmura et al., Gene amplification and overexpression of PRDM14 in breast cancers, Cancer Res. 67 (2007), 96499657.

[36] S. Nofech-Mozes, J. Spayne, E. Rakovitch and W. Hanna, Prognostic and predictive molecular markers in DCIS: a review, Adv. Anat. Pathol. 12 (2005), 256-264.

[37] P. O’Connell, V. Pekkel, S.A. Fuqua, C.K. Osborne, G.M. Clark and D.C. Allred, Analysis of loss of heterozygosity in 399 premalignant breast lesions at 15 genetic loci, J. Natl. Cancer Inst. 90 (1998), 697-703.

[38] F.P. O’Malley, S. Chia, D. Tu, L.E. Shepherd, M.N. Levine, V.H. Bramwell et al., Topoisomerase II alpha and responsiveness of breast cancer to adjuvant chemotherapy, J. Natl. Cancer Inst. 101 (2009), 644-650.

[39] K. Park, S. Han, E. Shin, H.J. Kim and J.Y. Kim, EGFR gene and protein expression in breast cancers, Eur. J. Surg. Oncol. 33 (2007), 956-960.

[40] J.S. Reis-Filho, S. Drury, M.B. Lambros, C. Marchio, N. Johnson, R. Natrajan et al., ESR1 gene amplification in breast cancer: a common phenomenon?, Nat. Genet. 40 (2008), 809-810.

[41] E.C. Robanus-Maandag, C.A. Bosch, P.M. Kristel, A.A. Hart, I.F. Faneyte, P.M. Nederlof et al., Association of C-MYC am- plification with progression from the in situ to the invasive stage in C-MYC-amplified breast carcinomas, J. Pathol. 201 (2003), 75-82.

[42] S.M. Rodriguez-Pinilla, R.L. Jones, M.B. Lambros, E. Arriola, K. Savage, M. James et al., MYC amplification in breast cancer: a chromogenic in situ hybridisation study, J. Clin. Pathol. 60 (2007), 1017-1023

[43] S. Sen, H. Zhou and R.A. White, A putative serine/threonine kinase encoding gene BTAK on chromosome 20q13 is amplified and overexpressed in human breast cancer cell lines, Oncogene 14 (1997), 2195-2200.

[44] J.F. Simpson, D.E. Quan, F. O'Malley, T. Odom-Maryon and P.E. Clarke, Amplification of CCND1 and expression of its protein product, cyclin D1, in ductal carcinoma in situ of the breast, Am. J. Pathol. 151 (1997), 161-168.

[45] M. Singh, M.J. Bleile, A.L. Shroyer, D. Heinz, E.A. Jarboe and K.R. Shroyer, Analysis of survivin expression in a spectrum of benign to malignant lesions of the breast, Appl. Immunohistochem. Mol. Morphol. 12 (2004), 296-304.

[46] P.J. van Diest, No consent should be needed for using leftover body material for scientific purposes, Br. Med. J. 325 (2002), 648-651.

[47] L. Wiechmann and H.M. Kuerer, The molecular journey from ductal carcinoma in situ to invasive breast cancer, Cancer 112 (2008), 2130-2142.

[48] Y. Zhu, C. Qi, S. Jain, M.M. Le Beau, R. Espinosa III, G.B. Atkins et al., Amplification and overexpression of peroxisome proliferator-activated receptor binding protein (PBP/PPARBP) gene in breast cancer, Proc. Natl. Acad. Sci. USA 96 (1999), 10848-10853. 


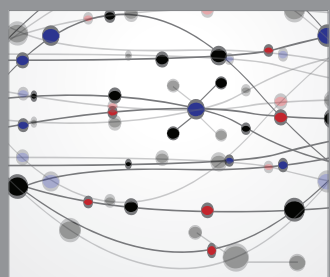

The Scientific World Journal
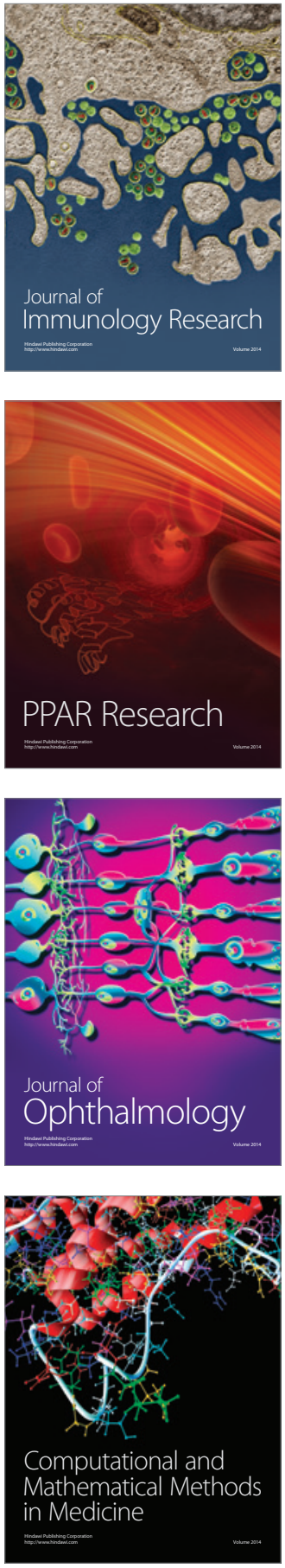

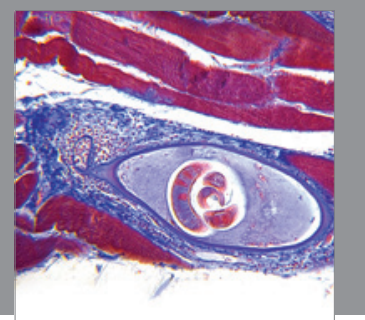

Gastroenterology

Research and Practice
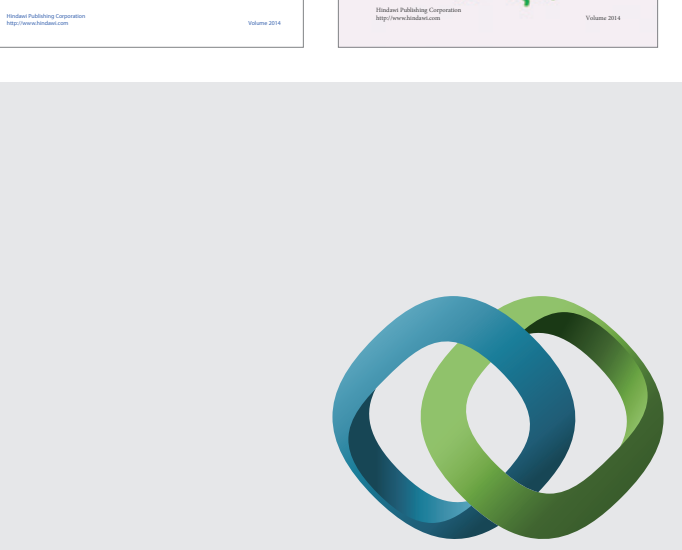

\section{Hindawi}

Submit your manuscripts at

http://www.hindawi.com
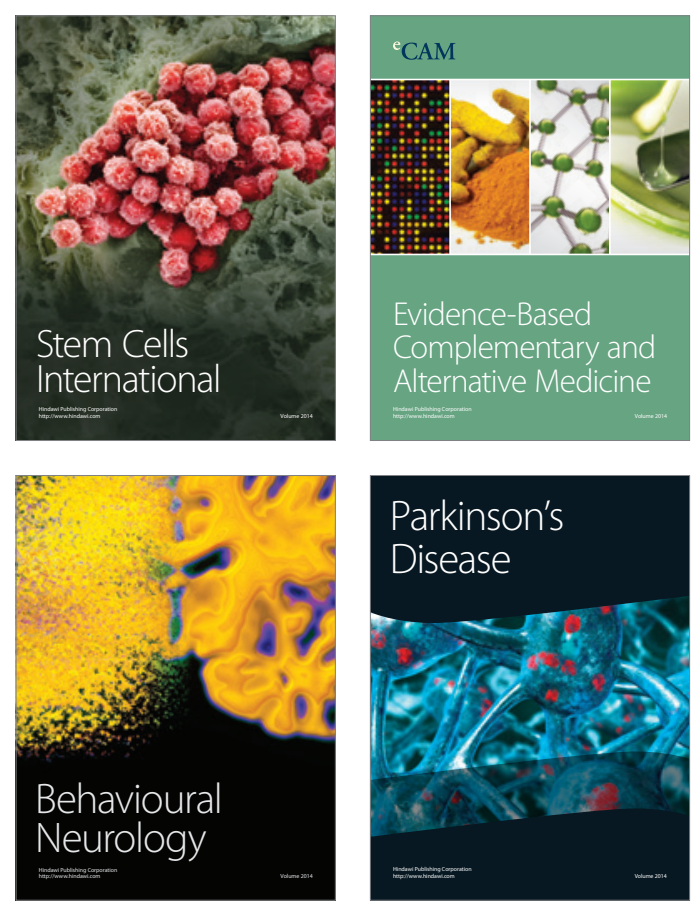

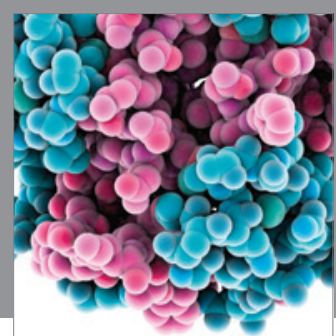

Journal of
Diabetes Research

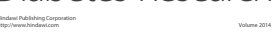

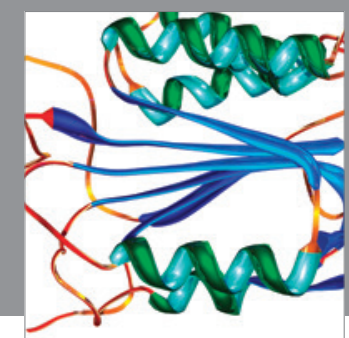

Disease Markers
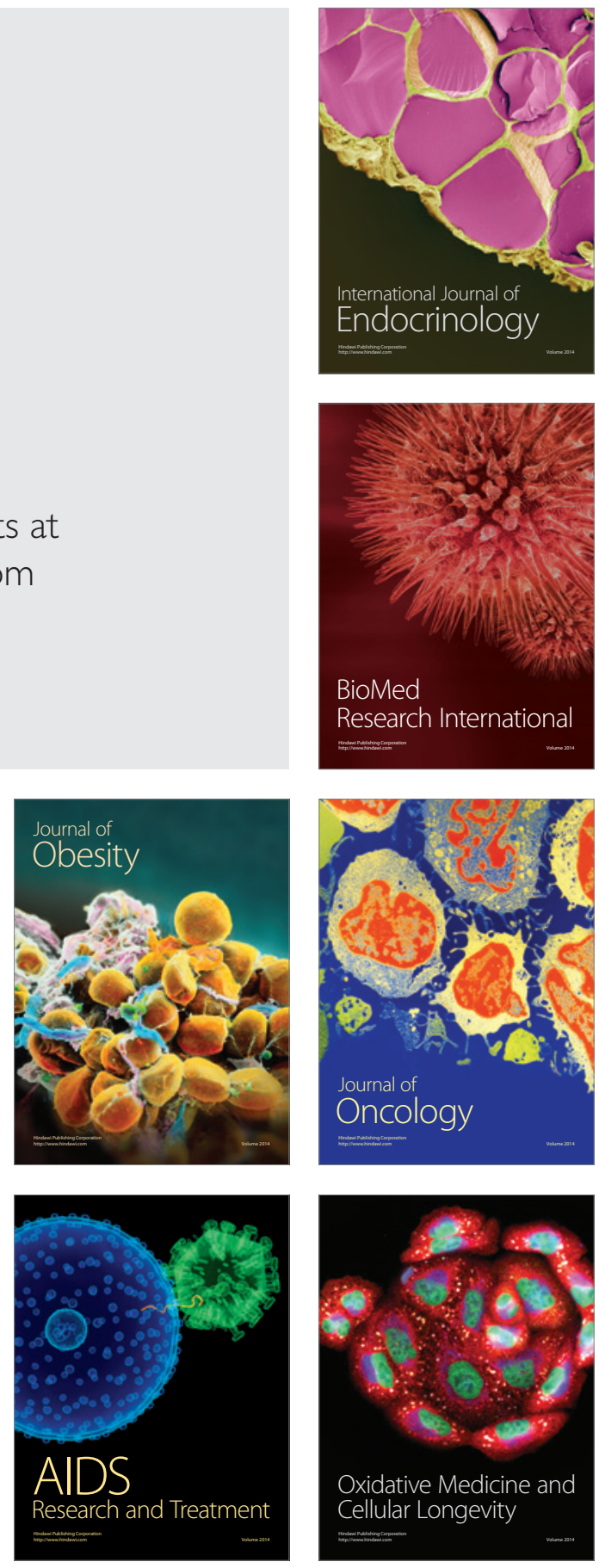